\title{
EFFECT OF NIMODIPINE ON HISTOLOGICAL ALTERATIONS IN BASILAR ARTERY FOLLOWING THE BILATERAL COMMON CAROTID ARTERY LIGATION (Preliminary Study)
}

\author{
Ümit Ö̈kan', Mehmet Dumlu Aydin'², Mustafa Serdar Kemaloglu' ${ }^{1}$ Fahri Yilmaz, Ismail Hakk Aydin ${ }^{4}$
}

Dicle University, School of Medicine, Diyarbakır, Turkey: Department of Neurosurgery ${ }^{1}$, Department of Pathology ${ }^{3}$; SSK Hospital, Erzurum, Turkey: Clinic of Neurosurgery2; Atatürk University, School of Medicine, Erzurum, Turkey: Department of Neurosurgery 4

Summary: Background: The blood supply to tissues is reduced as a result of arterial occlusions. Angiogenesis, collateral circulation and reverse flow mechanisms go into operation to restore a continued adequate supply of blood. Ca ${ }^{++}$channels undertake the major part of this function. As a result of the increasing tension on the arterial walls, vascular autonomy is affected, and ischemia and even necrosis are observed. Method: Adult 100 male hybrid rabbits were used in this study. The bilateral carotid arteries were ligated at the carotid bifurcation. The rabbits were divided into 2 main groups: treatment and control, and then both groups were further divided into 5 subgroups consisting of 10 rabbits each. The rabbits were sacrificed between the first day and the end of 8 weeks for histopathological examination of the basilar artery in two groups. Results: In control groups, after 24 hours of the occlusion partial swelling and minor endothelial damage were observed in histopathological sections of the basilar artery. Luminal flattening started to decrease, and expanding of the diameter continued. The increase in the diameters of the basilar artery was higher in animals treated by nimodipine, and that difference was statistically significant $(\mathrm{P}=0,000)$. Conclusion: This study revealed that the intimal and medial alterations arising from the increased blood flow rate in the basilar artery might be lessened and even partially prevented by the use of nimodipine.

Key words: Bilateral common carotid artery ligation; Basilar artery; Nimodipine

\section{Introduction}

The rate of blood flow is reduced as a result of arterial occlusion. In order to restore the rate of flow to the previous level, angiogenesis, collateral circulation and reverse flow mechanism move into action. Cranio-cerebral flow is lowered after the ligation of the bilateral common carotid artery. The vertebrobasilar system also bears the burden of the carotids by undergoing morphological and histopathological transformations to increase the rate of flow $(4,5$, $14,17)$.

New anastomosis are created with the neighbouring arterial structures. New shunts are established while the shunts in reserve are brought into operation. The blood is transferred through the reverse flow mechanisms from the vertebrobasilar system, which is the major blood supplier of the cranio-cervical region, to the external carotid artery and its branches (19). The other branches of the subclavian arteries also join this circulation and, cranio-cerebral circulation is eventually largely restored to its normal level $(19,24)$. The increased flow and volume cause some irritation and compression syndromes on the structure near the intracranial compartment, although it is nevertheless useful (10). This causes systemic complications in the brain and lungs $(6,11)$. In the basilar artery in particular, serious pathological situations may arise. Wall tension resistance constitutes the major obstacle to the required flow increase. The elastic and contractile units on the wall of the vessel receive the major part of the tension forces. It is believed that the vessel smooth muscle resistance may be overcome through the blocking of calcium channels.

The studies showed that dihydropyridine derivatives will help the organism after the ligation of the bilateral common carotid artery and support the survivor mechanisms with vasodilator flow increasing angioneogenesis, and its anti ischemic, perfusion regulatory, anti epileptic, cyto-protective and $\mathrm{pH}$ regulation effects $(7,8,12,16,18)$. 


\section{Materials and Methods}

Adult 100 male hybrid rabbits, weighing 3.0 to $3.5 \mathrm{~kg}$ each, were used in this study. All surgical procedures were performed with anaesthesia induced intramuscularly, using ketamine hydrochloride $(50 \mathrm{mg} / \mathrm{kg})$. The left femoral vein was catheterised for continuous infusion. Body temperatures and arterial blood pressure were frequently checked and maintained within the physiological ranges. The all rabbits were placed in the supine position with the head fixed to a horizontal orbitomeatal line. Using sterile technique, a midline cervical incision was made and the carotid arteries of all rabbits were bilaterally ligated at the carotid bifurcation. Only rabbits that were asymptomatic after carotid artery ligation were included in this study. All animals were kept on a standard diet and given water.

The rabbits were divided into 2 main groups: control and treatment, and then both groups were further divided into 5 subgroups each consisting of 10 rabbits. Group 1 served as the control, in which 50 rabbits received daily $0.2 \mathrm{ml}$ saline solution intravenously through the femoral catheter over 30 minutes by an infusion pump during the first 14 days after the bilateral common carotid ligation. In group 2 (treated group), 50 rabbits daily received $0.01-\mathrm{mg} / \mathrm{kg}$ nimodipine intravenously through the femoral catheter in $30 \mathrm{mi}-$ nutes by an infusion pump during the first 14 days after the bilateral common carotid ligation.

The all rabbits were sacrificed between the first day and the end of 8 weeks for histopathological examination of the basilar artery (at the end of the $1^{\text {st }}$ and $7^{\text {th }}$ days, and the $2^{\text {nd }}$, $4^{\text {th }}$ and $8^{\text {th }}$ weeks for each subgroups).

The rabbits' brains were removed immediately, and the specimens were then placed in a $10 \%$ formaldehyde solution for five days. After fixation of the tissues, the samples were dehydrated using a graded ethyl alcohol series and cleared through xylen. Tissues were transferred to an incubator $\left(30^{\circ} \mathrm{C}\right)$, and each was processed in two different paraffin baths, $\left(56{ }^{\circ} \mathrm{C}\right)$ for 30 minutes. Following paraffin embedding, 8 ( $\mathrm{m}$ sections were cut by a steel blade using a rotator microtome. Tissue slices were floated on albumined slides and allowed to dry for 12 hours at $40{ }^{\circ} \mathrm{C}$. Routine staining was performed using Harris' Hematoxylene counter and alcoholic eosin. The samples were examined and photographed using an Olympus microscope.

Planimetric measurements were made via an oculometer at x100 magnification of light microscopy. Luminal crosssectional diameters of the rabbit basilar artery were measured. The planimetric measurements were made on a cross-sectional diameter of the basilar artery. The diameter of the basilar artery was measured in specimens at three locations: near vertebrobasilar junction, midpoint and close to basilar tip. Vertical and horizontal diameters of the basilar artery were measured, and the median value of these measurements accepted as the true diameter for each section. Two different pathologists performed the measurements independently in blind conditions, and the average value thus obtained was used in our study.

Three slices were taken from each specimen for planimetric and histological analysis. Data for each animal were expressed as a percentage change for the experimental versus the control artery, and were pooled for analysis according to the indices listed. Differences between groups were assessed with the 2-tailed t-test, and the results expressed as mean and standard deviation (Tab. 1).

\section{Results}

Histopathological examination with H\&E staining showed narrowing in the cross-sectional diameter of the vascular lumen and vascular wall in both the control and treatment groups. Endothelium formed a continuous monolayer overlying a thin, undulated internal elastic lamina. Five to eight layers of concentrically oriented smooth muscle cells surrounded the intima. The normal structure of the basilar artery is shown below (Fig. 1).

In the control groups, 24 hours after occlusion partial swelling and minor endothelial damage were observed during histopathological examination of the basilar artery (Fig. 2). In the treated groups, 24 hours after occlusion the appearance of the basilar artery and endothelium was largely intact, the lumen was flattened and vessel muscles began to elongate (Fig. 3). Endothelial damage was more

Tab. 1: Diameters of rabbits basilar artery in both control and treated groups.

\begin{tabular}{|lc|cc|cc|}
\hline \multirow{2}{*}{ Experiment } & \multicolumn{4}{c|}{ Diameters of basilar artery } \\
Course Time & t value & \multicolumn{2}{c|}{ Control groups (Micron) } & \multicolumn{2}{c|}{ Treated groups (Micron) } \\
\hline $\mathbf{2 4}$ Hours* & $\mathbf{2 6 , 6 3 1}$ & M: $766 \pm 0.01$ & SD: 12,05 & M: $906 \pm 0.15$ & SD: 11,44 \\
\hline 7 Days** & $\mathbf{2 1 , 6 7 7}$ & M: $812 \pm 0.02$ & SD: 16,89 & M: $1000 \pm 0.20$ & SD: 21,70 \\
\hline 14 Days* $^{\text {4 Weeks }}$ & $\mathbf{2 1 , 3 2 7}$ & M: $886 \pm 0.03$ & SD: 12,36 & M: $1300 \pm 0.3$ & SD: 60,16 \\
\hline 8 Weeks & $\mathbf{1 , 3 8 2}$ & M: $1045 \pm 0.02$ & SD: 60,05 & M: $1080 \pm 0.02$ & SD: 53,32 \\
\hline
\end{tabular}

\section{M: Mean; SD: Std. Deviation}

The increase in the diameters of the basilar artery was higher in animals treated by nimodipine, and that difference was statistically significant [2-tailed t-test], * $\mathrm{P}=0,000$ 
evident on the $7^{\text {th }}$ day than on the first, and arterial wall thickness was thinner than on the first day. After the second week endothelial proliferation was observed. Furthermore, lumens became more flattened and the wall continued to grow thinner. In the control group, the sinusoidal structure of the membrana elastica externa changed to a linear form by the $14^{\text {th }}$ day (Fig. 4).

In the treated groups, an elongation in the vessel muscles, an excessive widening in the lumen, local intimal and medial destruction were seen after 14 days (Fig. 5). At the end of the first month, endothelial damage decreased while luminal flattening and membrana elestica externa elonga-

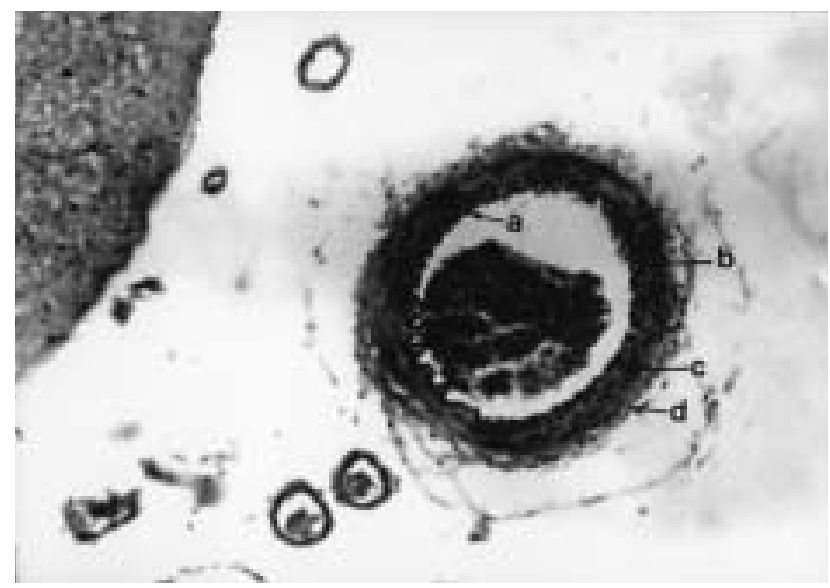

Fig. 1: Normal basilar artery: Endothelium (a), Membrana elastica externa (b), Tunica media (c), Adventitia (d). There is no membrana elastica interna in the vessels of the brain. (LM-H\&E, x100)

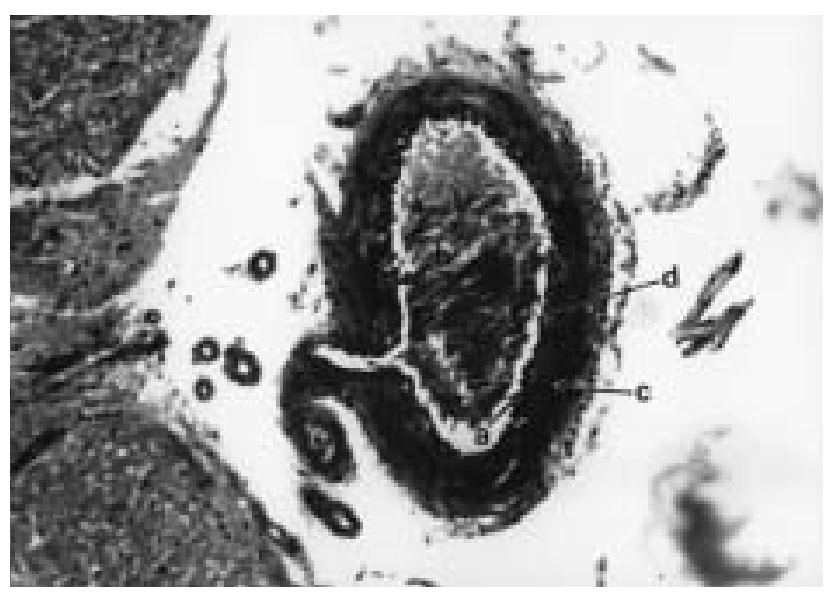

Fig. 2: The appearance of the basilar artery the day after the bilateral common carotid artery ligation: There is widening in the arterial lumen (L) together with a swelling in the endothelium (a), a minimal destruction (b), an intimal oedema (c), and local endothelial proliferations (d). (LM-H\&E, x100) tion increased. During the course of the experiment, the luminal diameter of the basilar artery increased and the membrana elastica externa regained its own sinusoidal structure.

At the end of the second month in the control groups, significant intimal proliferation and the presence of increased medial matrix, smooth muscle hypertrophy and an excessive indentation in the membrana elestica externa were seen. Luminal flattening started to decrease, and expansion of the diameter continued (Fig. 6).

In the treatment groups, nimodipine prevented endothelial swelling, decreased the damage that occurred over

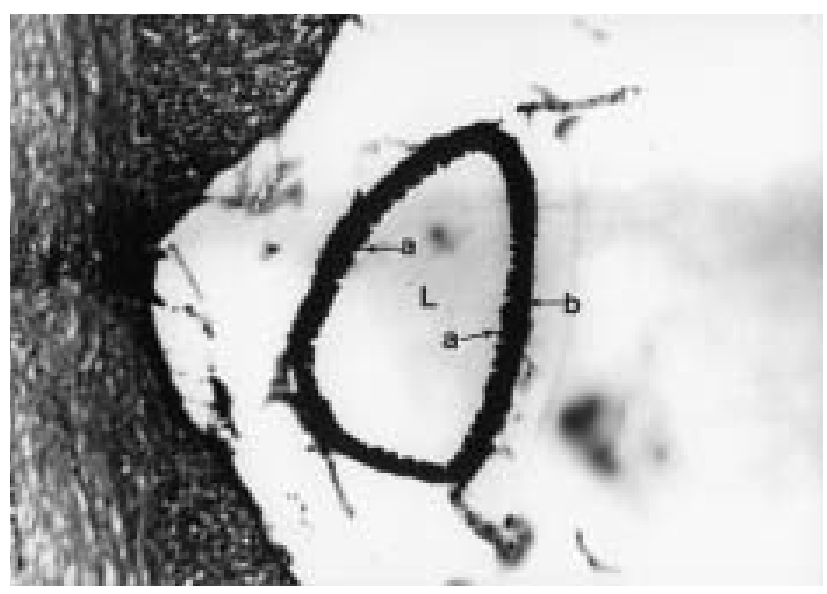

Fig. 3: The appearance of basilar artery in the animal which received nimodipine after bilateral common carotid artery ligation : The endothelium is basically intact and (a), the lumen is flattened $(\mathrm{L})$ and the vessel muscles have become smooth (b), (LM-H\&E, x100)

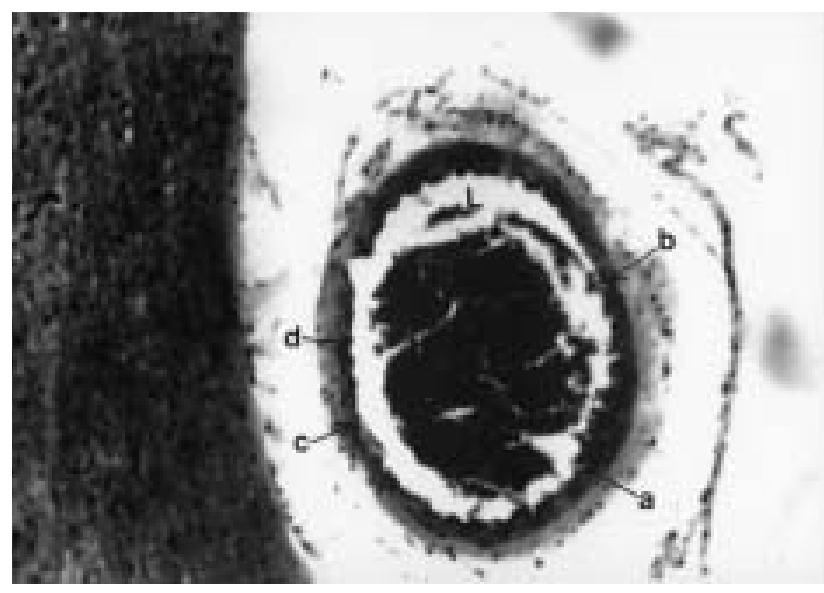

Fig. 4: The appearance of the basilar artery 14 days after the bilateral common carotid artery ligation: there is a proliferation in the endothelium (a), to become thin in the wall (b), an expansion in the lumen (L), an elongation in the smooth muscles (c), external elastic membrane (d). (LMH\&E, x100) 


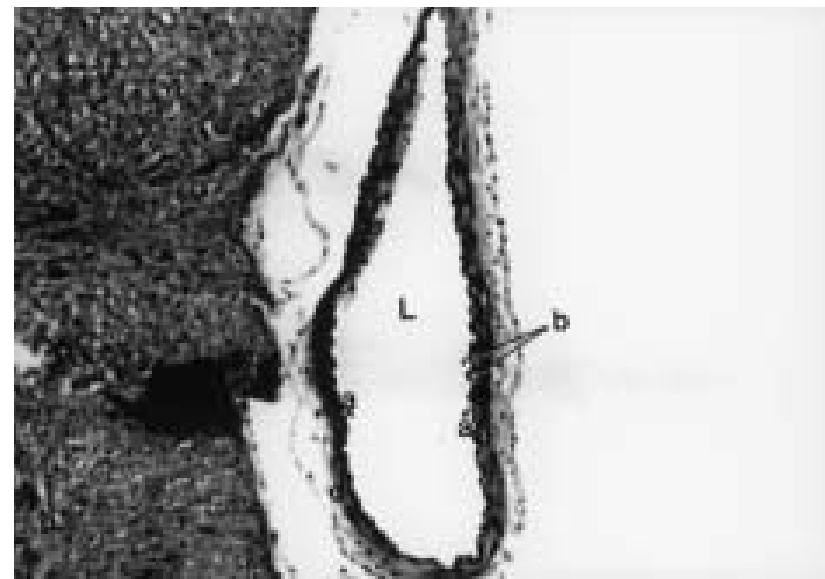

Fig. 5: The appearance of the basilar artery 14 days after the bilateral common carotid artery ligation together with the infusion of nimodipine: The endothelium has a healthy appearance (a), An elongation in the vessel muscles and (b) excessive widening in the lumen. There are local intimal (c), and medial (d), destruction. (LM-H\&E, x100)

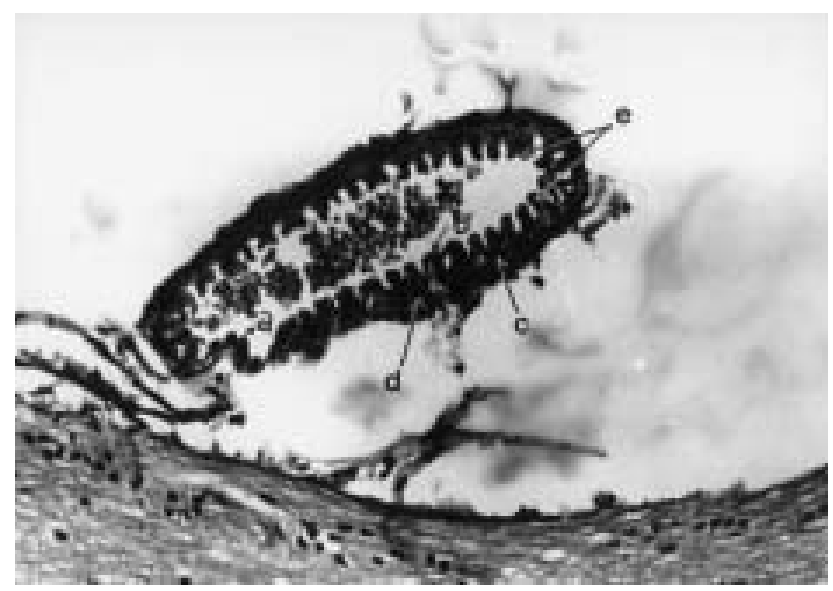

Fig. 6: The appearance of the basilar artery two months after the bilateral common carotid artery ligation: The endothelium has a normal appearance (a), membrana elastica externa is locally normal (b), there is a smooth muscle hypertrophy(c), an intimal proliferation and (d), an excessive indentation in the membrana elastica externa (e). (LM$\mathrm{H} \& \mathrm{E}, \mathrm{x} 100)$

the course of time and protected the endothelial cells (Fig. 5). Its relaxing effect was clearly apparent on the smooth muscles in the first and second weeks. Muscles started to regain their convoluted structures after it was withdrawn. The nimodipine significantly prevented the intimal proliferation and medial matrix towards the end of the second week. At the end of the second month, therefore, endothelial structure regained its normal form and the wall thickness returned to normal. The membrana elastica externa

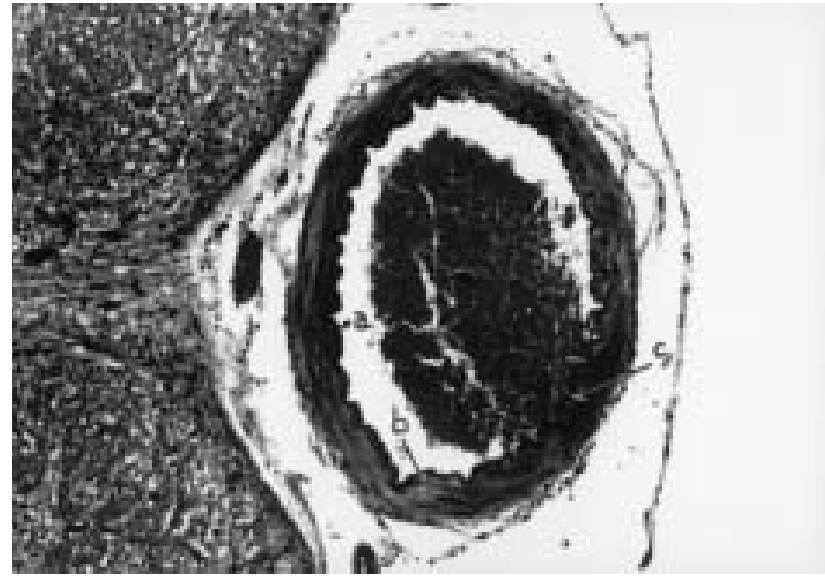

Fig. 7: The appearance of the basilar artery two months after the bilateral common carotid artery ligation together with infusion of nimodipine: The artery appears quite normal, while the diameter is still above the normal value ( $\mathrm{a}$ - endothelium, b - membrana elastica externa, c - smooth muscles). (LM-H\&E, x 100)

partially reverted to its normal shape. The lumen had become circular, and the diameter was still above the normal value (Fig. 7).

In our study, we noticed a gradual increase in the diameters of the basilar artery as time progressed. The increase in the diameters of the basilar artery was higher in animals treated by nimodipine, and that difference was statistically significant ( $\mathbf{P = 0 , 0 0 0}$ ) (Tab. 1). As the diameters increased, deceleration were observed after the second month because of the reduced blood load on the vertebrobasilar system as collateral circulation came into effect, and after the third week because of a decrease in the vasodilator forces as the nimodipine was withdrawn. At the end of the forth weeks, the values that we obtained were lower than the maximum values. The nearing of normal limits of the lumen may be attributable to the membrana elastica externa and muscular activity at the end of the fourth weeks, but there is no such mechanism that might lead to the reduction of length.

\section{Discussion}

The bilateral ligation of Common Carotide Artery results in an active reorganisation in all parts of the craniocerebral and cervical arterial system, in addition to the vertebrobasilar system, accompanied by major hemodynamic alterations. This phenomenon causes new formations in carotico-vertebrobasilar structures and important hemodynamic changes at the beginning of the reverse flow mechanisms. As a result of these hemodynamic changes, blood is transmitted from posterior to anterior circulation by the help of normal shunts (circulus of Willis'). In fact, there can be flow from Arteria Carotis Interna to Arteria Carotis Externa and Cerebral Blood Flow and a return to 
normal within a time span of two months as a result of these phenomena. It is reported that the mortality rate is around $21 \%$ in experimental bilateral carotid occlusion. It is shown that cerebral oedema and herniations cause high mortality, especially in the first days $(4,6)$. In our study, the mortality rate was $9.9 \%$ in the control group, $4.4 \%$ in the test group and $14.3 \%$ in total.

In the treated group microscopic examination indicated that nimodipine delayed and reduced the endothelial damage that appeared in the control group on the first day and the seventh. A similar situation was also observed by Allen (1) et al. Luminal flattening and wall have become thin in the basilar artery, which appeared because of the extension of periods after the ligation and continued until the second month, are indicated by Oldendorf and his colleagues (19). Luminal flattening and extension in the muscles of the vessels was high in the nimodipine group, and luminal widening was proved both radiologically and histologically. Furthermore, the vasodilatatory effects of nimodipine was shown radiologically by Findlay, and by Auer and Kazda histopathologically $(2,9,13)$.

Endothelial proliferation started after the second week. No endothelial proliferation was encountered in those subjects which received nimodipine. This observation support the findings of the paper regarding the anti-proliferative effect of nimodipine (1). In the second week, the intimal and medial damage has not appeared in those subjects who received nimodipine. It is assumed that it is an important mediator for cellular activities and a major element in mitosis (20). There is also the possibility that the increased intercellular distance in the arteries and the muscular support had weakened or may have severed the intercellular bonds. This result confirms that intermittent use of nimodipine is likely to be beneficial. The membrana elastica externa that began to flatten in the first weeks of the study, started to regain its original structure after the second month. These changes were greater in those individuals receiving nimodipine. An elongation may be expected as a result of the flattening in the membrana elastica externa that is unable to respond adequately to the vascular resistance as result of the inhibition of vessel smooth muscles by nimodipine. Afterwards, the endothelial flattening received particular attention.

Lehman and his colleagues measured the normal diameters of the basilar artery in rabbits as $0.761+0.004 \mathrm{~mm}$ (15) in the post-mortem period, but the values which we obtained were $0.765+0.005 \mathrm{~mm}$. The standard deviations that appeared during the experiment displayed a similar trend.

Increased blood flow forces and a likely decrease in Growth Inhibition Factor (GIF) may have played an important role in the appearance of morphological and histological changes in the arterial system we were examining $(19,21)$. It is reported that NO which enters into action as a result of partial hypoxia may have had an effect in the early period of widening $(16,23)$. The significant vasodilatation that was observed in the test group can be considered a result of the vasodilator effects of nimodipine $(3,10,22)$. Vasodilatation started to recede as the nimodipine was withdrawn. This phenomenon may be based on the vasodilator effect on vessel plain muscles. However the average diameter of this group was higher than the other group in the following period as well. This result may tend to indicate that the collaterals were still dominant and that the blood flow to these regions was still adequate.

From the middle of second month onward, increases were observed in the formation of medial matrix and intimal proliferation. Several researchers had mentioned these changes $(17,19)$. This phenomenon was not encountered in those animals which had taken nimodipine. In fact, other researchers also confirm that nimodipine prevented this phenomenon $(1,13)$.

In conclusion, the increase in the diameters of the basilar artery was higher in those individuals which had taken nimodipine, and that difference was statistically significant. As the diameters increased, deceleration were observed after the second month because of the reduced load on the vertebrobasilar system as collateral circulation came into effect, and after the fourth weeks because of a decrease in the vasodilator forces as the nimodipine was withdrawn. This situation is suggestive of a probable relationship between calcium and the Growth Inhibition Factor (GIF) mechanism. However, further work is necessary to confirm these preliminary findings and to clearly delineate the mechanisms of action.

\section{References}

1. Allen GS, Ahn HS. Cerebral arterial spasm-a controlled trial of nimodipine in patients with subarachnoid hemorrhage. N Engl J Med 1983;308:619-24.

2. Auer LM. Cerebrovascular activity of the calcium antagonist nimodipine. J Cereb Blood Flow Metab 1982;2:268-73.

3. Badesch DB, Lee PDK et al. Insulin-like growth factor-1 stimulates elastin synthesis by bovine pulmonary arterial smooth muscle cells. Biochem Biophys Res Commun 1989;160:382-7.

4. Catteral WA. Structure and function of voltage-sensitive ion channels. Science 1988;242:50-61.

5. Conrad MC, Anderson JL III, Garrett JB. Chronic collateral growth after femoral artery occlusion in the dog. J Appl Physiol 1971;31:550-5.

6. Edvinsson L, Emson $\mathrm{P}$ et al. Neuropeptide Y: cerebrovascular innervation and vasomotor effects in the cat. Neurosci Lett 1983;43:79-84.

7. Eklöf B, Siesjö BK. The effect of bilateral carotid artery ligation upon the blood flow and energy state of the rat brain. Acta Physiol Scand 1972;86:155-65.

8. Feng P, Ohlsson M.N T. The structure of the TATA-less rat tissue-type plasminogen activator gene. Species-specific sequence divergences in the promoter predict differences in regulation of gene expression. J Biol Chem 1990;265:2022-7.

9. Findlay JL, Macdonald RL, Weir BK. Current concepts of pathophysiology and management of cerebral vasospasm following aneurysmal subarachnoid hemorrhage. Cerebrovasc Brain Metab Rev 1991;3:336-61.

10. Haws CW, Heistad DD. Effect of nimodipine on cerebral vasoconstrictor responses. Am J Physiol 1984; 247(2 Pt 2) Ph:170-6.

11. Iwasaki $\mathrm{Y}$, Ito $\mathrm{S}$ et al. Forebrain ischemia induced by temporary bilateral common carotid occlusion in normotensive rats. Neurol Sci 1989;90:155-65.

12. Kazda S, Garthoff B et al. Cerebrovascular effect of the calcium antagonistic dihydropyridine derivative nimodipine in animal experiments. Arzneimittelforschung 1982;32:331-8

13. Kazda S, Tovard R. Nimodipine: a new calcium antagonistic drug with a preferential cerebrovascular action. Acta Neurochir 1982;63:259-65.

14. Langille BL, O'Donnel F. Reductions in arterial diameter produced by chronic decreases in blood flow are endothelium dependent. Science 1986;231:405-7.

15. Lehman RM, Owens GK. Mechanism of enlargement of major cerebral collateral arteries in rabbits. Stroke 1991;22(4):499-503.

16. Meyer FB, Anderson RE. Selective central nervous system calcium channe blockers-a new class of anticonvulsant agents. Mayo Clin Proc 1986;61(4):239-47. 
17. Mignatti $\mathrm{P}$, Tsuboi R. Invitro angiogenesis on the human amniotic membrane requirement for BFGF-induced proetinases. J Cell Biol 1989;108:671-82.

18. Mohamed AA, Gotoh O et al. Effect of pretreatment with the calcium antagonist nimodipine on local cerebral blood flow and histopathology after middle cerebral artery occlusion. Ann Neurol 1985;18:705-11.

19. Oldendorf WH. Trophic changes in the arteries at the base of the rat brain in response to bilateral common carotid ligation. Neuropath and Exp Neurol 1989:48(5):534-47.

20. Robinson MJ, Teasdale GM. Calcium antagonists in the management of subarachnoid haemorrhage. Cerebrovasc Brain Metab Rev 1990;2:205-26.

1. Rodbard S. Vascular caliber. Cardiology 1975;60:4-49.

22. Rosenfeld MG, Mermod JJ et al. Production of a novel neuropeptide encoded by the calcitonin gene via tissue-specific RNA processing. Nature 1983;304:129-35.

23. Strandness DE Jr. Biology of the collateral circulation. In: Schwartz CJ, Wethessen NT, Wolf S. Structure and function of the circulation. New York: Plenum press 1980;1:487-535.

24. Truchiya M, Kazuhiro Sako. Cerebral blood flow and histopathological changes following permanent bilateral carotid artery ligation in Wistar rats Exp Brain Res 1992;89:87-92.

Submitted July 2003.

Accepted November 2003.

Dr. Ümit Özkan,

Dicle University Faculty of Medicine,

Department of Neurosurgery

21280 Diyarbakır, Turkey.

e-mail: drumitozkan@yahoo.com 